\title{
Probing electronic radial wave packets using impulsive momentum retrieval
}

\author{
M. B. Campbell, T. J. Bensky, and R. R. Jones \\ Department of Physics, University of Virginia, Charlottesville, Virginia 22901
}

\begin{abstract}
Subpicosecond half-cycle pulses and a single-shot imaging detector have been used to monitor the evolution of electronic radial wave packets in calcium. The time-dependent momentum-space probability distribution is obtained using the impulsive momentum retrieval (IMR) method. The measured distributions are compared to the results of quantum and classical simulations allowing a comprehensive evaluation of the benefits and limitations of the IMR method.
\end{abstract}

\section{INTRODUCTION}

During the past decade, rapid technological progress has made it possible to create and probe a variety of different coherent superposition states, or wave packets, in atoms [1]. Understanding the dynamic evolution of these nonstationary states is important to studies of classical-quantum correspondence, the response of atoms to strong static and dynamic electromagnetic fields, and quantum control in atoms and molecules. Experimentally, the problem of how to accurately probe electronic dynamics has proved to be as difficult as, if not more difficult than, producing the wave packet in the first place [2]. The techniques of short pulse pump-probe ionization [3], bound-state interferometry [4], and time-resolved streak camera detection of ionization [5] have all been used to characterize wave packet evolution with varying degrees of success. However, none of these methods are useful for determining the time-dependent electronic probability distribution.

Recently, two methods have been developed that make it possible to measure, approximately, the projection of the probability distribution of an electronic wave packet on one coordinate axis. The first, time-resolved isolated core excitation (ICE) [6], relies on electron correlation in multielectron atoms to determine the position of one electron during the optical excitation of another, tightly bound electron [7]. The second, impulsive momentum retrieval (IMR), utilizes ultrashort, unipolar field pulses (HCPs) to determine one Cartesian component of the momentum space probability distribution of a wave packet [8]. Although these two methods are quite straightforward in concept, they are both extremely difficult to implement in practice. Both require that data be collected to fill a two-dimensional parameter space. For example, the IMR method requires a measurement of wave packet ionization probability versus HCP field amplitude versus delay [8]. The time required to make these measurements is often so great that unavoidable equipment drifts make an accurate determination of the probability distribution impossible.

To circumvent this problem, we have constructed a detector that makes it possible to collect time delay data in real time using a single laser shot [9]. As a result, the timeresolved ICE or IMR procedures can be reduced to a scan over a single parameter. This paper describes the use of this "single-shot detector" and the IMR method in the measure- ment of the time-dependent momentum distribution of a radial electronic wave packet. In the following sections we describe our experimental method and compare the experimental results to those of theoretical simulations. We conclude with a discussion of the applicability of the method to future experiments.

\section{EXPERIMENTAL PROCEDURE}

Atoms in the $4 s 4 s{ }^{1} S_{0}$ ground state in a thermal Ca beam are pumped to the $4 s 4 p{ }^{1} P_{1}$ level using 423-nm radiation from a 5-nsec dye laser pulse. The intermediate state atoms are further excited to a linear superposition of $4 s n d{ }^{1} D_{2}$ Rydberg states using a 0.5-psec, 392-nm laser pulse. The 0.5 -psec laser pulse is generated via second-harmonic generation of the 120-fsec, 786-nm output from an amplified Kerr lens mode-locked Ti:sapphire laser. Phase-matching effects in the 1-cm-long potassium dihydrogen phosphate (KDP) doubling crystal restrict the bandwidth of the 392-nm pulse to approximately $30 \mathrm{~cm}^{-1}$, increasing its duration to approximately four times that of the fundamental. Since the lasers are all linearly polarized in the vertical $\hat{z}$ direction, the $z$ projection of the orbital angular momentum of the $n d$ Rydberg electron is restricted to $m=0$. Using state-selective field ionization [10], we determine that the radial wave packet contains appreciable amplitude from approximately seven 4 snd states centered about a mean principal quantum number $N=32.9$.

After its creation, the wave packet is exposed to a unipolar HCP. The HCP is created by gating a GaAs photoconductive switch with a portion of the 120-fsec, 786-nm Ti:sapphire output [11]. The HCP field amplitude is linearly proportional to the bias voltage on the switch and its polarization direction is parallel to the bias field [11]. The switch is mounted on a rotation stage so that the HCP polarization can be rotated continuously about its propagation direction. Before reaching the Rydberg atoms, the HCP propagates through an unbiased GaAs wafer. The reflectivity of this wafer is gated by an additional 120-fsec, 786-nm laser pulse producing a transient mirror that significantly attenuates any nonunipolar components of the HCP from reaching the Rydberg atoms $[11,12]$.

After passing through the transient attenuator, the duration of the "cleaned" HCP is approximately 1-psec. The fundamental oscillation period of the wave packet $2 \pi N^{3}$ a.u. 


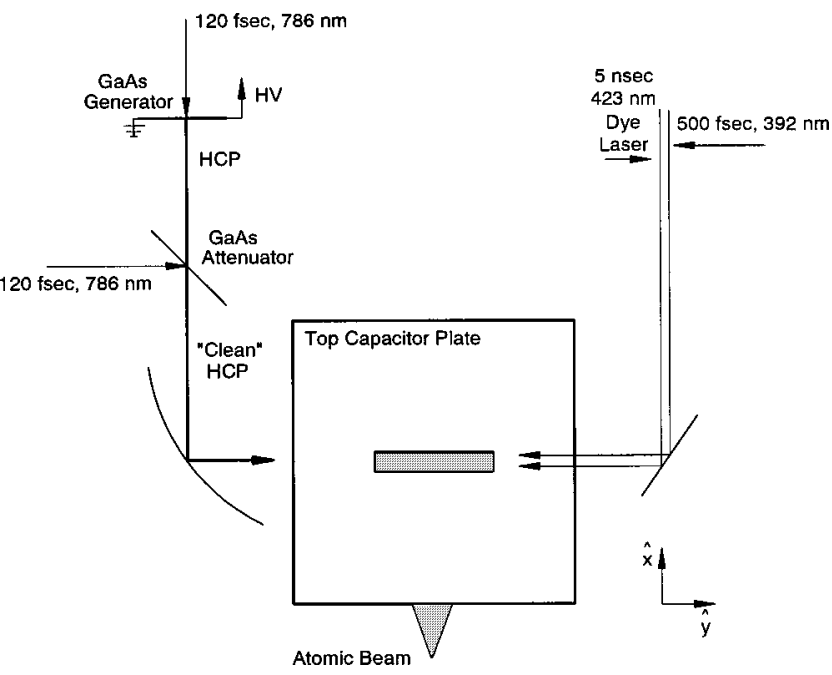

FIG. 1. Schematic of the laser-atom interaction region showing the relative orientation of the laser and atomic beams, the high voltage (HV) biased GaAs wafer, and the extraction slit.

$\approx 5.4 \mathrm{psec}$ is significantly longer. Therefore, to a first approximation, it is not unreasonable to consider the interaction of the HCP and the Rydberg electron to be impulsive. In this short-pulse approximation, the probability for ionizing the wave packet with a HCP depends only on the impulse delivered by the pulse (proportional to the time-integrated HCP field) and the momentum distribution of the wave packet just before the pulse $[13,14]$. Following the IMR procedure outlined in Ref. [8], the projection of the wave packet's momentum distribution along the HCP polarization direction can be obtained at any instant by measuring the ionization probability versus HCP field amplitude. The time-dependent momentum distribution is constructed by collecting ionization versus HCP field data at different relative delays between the 392-nm laser pump and HCP probe. The distributions along orthogonal axes are obtained by making identical ionization measurements with the HCP polarized in an orthogonal direction.

Using a different detector, the time dependence of the ionization probability at a fixed HCP field can be obtained with a single shot of the laser system [9]. With this device, the data required to span the two-dimensional parameter space, ionization versus HCP field versus delay, are collected in a single scan of the HCP field amplitude. The principle of operation behind the single-shot detector is similar to that of optical cross correlators that are commonly used to measure the temporal profile of ultrashort laser pulses. As shown in Fig. 1, the laser pump and HCP probe beams counterpropagate along the $\hat{y}$ direction and at right angles to the atomic beam so that all three beams lie in a common horizontal plane. The width of the atomic beam along the $\hat{y}$ axis is approximately $2.5 \mathrm{~cm}$. Because the pump and probe beams counterpropagate, the relative delay between the laser and $\mathrm{HCP}$ varies linearly along the $\hat{y}$ axis. The difference in the relative delay between the HCP probe and 392-nm pump as "seen" by two atoms separated by a distance $d$ along the $\hat{y}$ axis is $\Delta \tau=2 d / c$, where $c$ is the speed of light. Therefore, atoms that experience a continuous range of pump-probe delays are produced with each laser shot and the number of

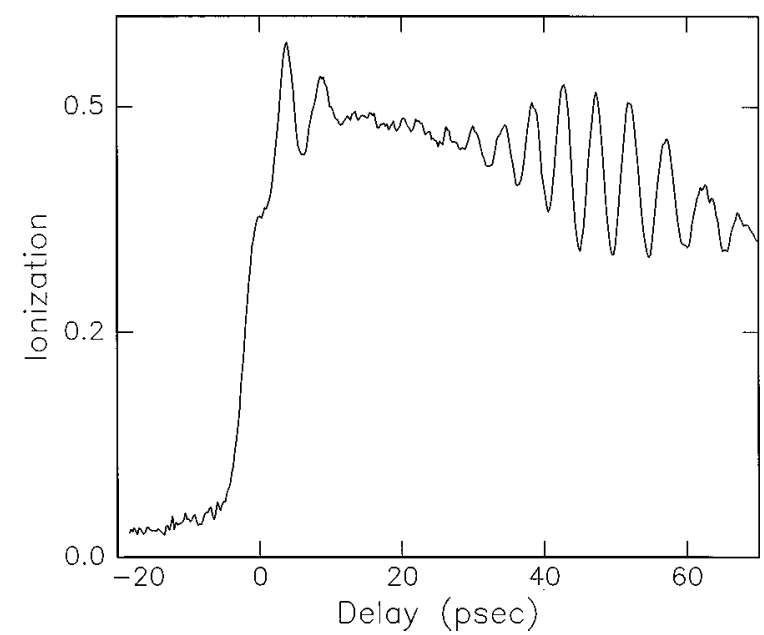

FIG. 2. Probability for ionizing the 4 snd wave packet as a function of time. The curve is the average of 300 single-line traces from the imaging detector.

ions produced as a function position along the $\hat{y}$ axis reflects the ionization probability as a function of HCP delay.

The laser, HCP, and atomic beams cross between two $7.5 \times 7.5 \mathrm{~cm}^{2}$ parallel capacitor plates that are separated by $1.5 \mathrm{~cm}$. The long dimension of a $0.15 \times 2.5 \mathrm{~cm}^{2}$ slit is oriented parallel to the $\hat{y}$ axis in the upper capacitor plate. Approximately $20 \mathrm{nsec}$ after the HCP interacts with the atoms, an approximately $50 \mathrm{~V}$ pulse applied to the lower field plate pushes any ions formed by the HCP through the slit toward a microchannel plate (MCP) detector. The ions strike the channel plates with their relative positions along the $\hat{y}$ axis preserved. The secondary electrons formed in the channel plates are accelerated onto a phosphor screen mounted to the top of the channel plate assembly. The spatial distribution of ions in the interaction region can be determined after each laser shot by monitoring the phosphor fluorescence level with a charge coupled device (CCD) camera. The spatial resolution of the combined phosphor and MCP is approximately $300 \mu \mathrm{m}$. Therefore, in this geometry, the ionization probability over a 100-psec HCP delay range can literally be seen with 1-psec resolution on a television monitor after a single shot.

Due to the finite width of the ion extraction slit in the upper field plate, approximately 40 lines in the video image contain ionization versus time-delay information. Although integrating the signal from each line enhances the signal-tonoise ratio and reduces the data collection time, it is not possible with our current computer interface to collect all of the useful video image and process it at the $15-\mathrm{Hz}$ laser repetition rate. Therefore, a digital oscilloscope is used to capture a single line of the video signal at the laser repetition rate. This line image is averaged over several hundred laser shots to improve the signal-to-noise ratio. Figure 2 shows the probability for ionizing a $4 s n d$ wave packet acquired from a single video line averaged over 300 laser shots $(20 \mathrm{sec}$ of data collection).

Spatial nonuniformities in the microchannel plate and phosphor efficiency are removed by normalizing the output of each CCD pixel individually. The normalization factor for each pixel is equal to its output voltage when the atoms are exposed to a HCP capable of ionizing the wave packet with 


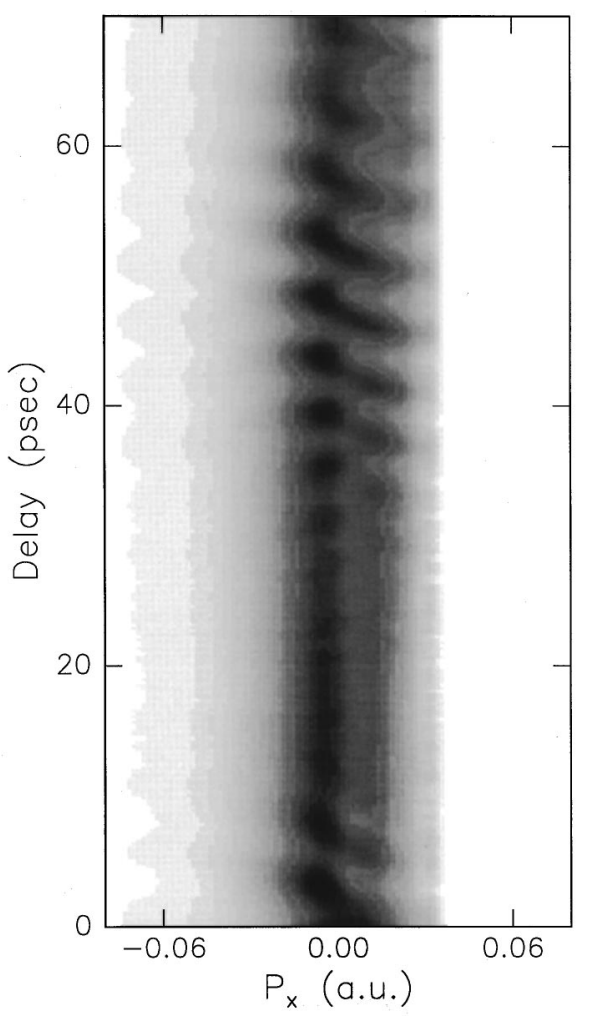

FIG. 3. Density plot of the experimental $p_{x}$ momentum distribution for a radial wave packet measured using the IMR method. The measured distribution for $p_{z}$ is similar but not shown due to technical difficulties as discussed in the text. Dark regions indicate high probability. Note the clear oscillations in the width of the distribution as the wave packet oscillates radially.

$100 \%$ efficiency. In addition, because of slight ion focusing by the extraction slit, the conversion from spatial position to delay is not perfectly linear. Therefore, the delay axis is calibrated using a quadratic function that describes the change in the observed spatial ionization distribution as the optical path length of the 392-nm pump pulse is varied.

Previous measurements [11], indicate that the peak field of the HCP is linearly proportional to the bias voltage on the photoconductive switch while its duration is independent of this voltage. Therefore, the impulse delivered by the HCP (i.e., the time-integrated field) is equal to the measured switch bias multiplied by a constant scaling factor. Taking advantage of the agreement of previous ionization experiments with classical calculations $[8,13,15]$, we assume that the impulse delivered by the HCP at $50 \%$ ionization is simply $A=1 / N$ a.u., where $A$ is the impulse or time-integrated $\mathrm{HCP}$ field and $N$ is the average principal quantum number of the wave packet.

To measure momentum distributions, ionization probability versus time scans similar to the one in Fig. 2 are collected at 50 different HCP field values. Each time scan is discretized into 300 data points and the data are resorted to give ionization probability versus field curves at 300 different delay times. The time-dependent momentum distribution is obtained by taking the derivative of a spline fit to the ionization versus field (or impulse) data at each time delay. Figure 3 shows the measured distribution of probability along the $p_{x}$ coordinate for the 4 snd radial wave packet. The symmetry of the atoms ensures that the distribution along $p_{y}$ is identical to that of $p_{x}$. Surface reflections of the HCP from the transient attenuator (prior to its illumination) reduce the $\mathrm{HCP}$ amplitude significantly. Therefore, we cannot determine the momentum distribution at large positive momentum where large impulses are required. Nevertheless, the timedependent distribution clearly reflects the anticipated motion of the wave packet.

At a delay $\tau=0$, the wave packet is located near the nucleus where there is a high probability that the magnitude of its momentum is very large. The momentum distribution narrows as the wave packet moves away from the nucleus as time evolves. The distribution becomes a narrow peak centered about zero momentum as the wave packet nears the outer turning point of the classical motion. The wave packet returns to the nucleus after a delay equal to the classical Kepler period $\tau_{K}=\approx 5.4 \mathrm{psec}$. The return is characterized by a dramatic broadening of the momentum distribution. Due to the anharmonicity of the Coulomb potential, the wave packet spreads and collapses after only two oscillations. Fractional revivals of the wave packet occur, but they cannot be seen due to the limited resolution of the detector. However, a full revival of its momentum oscillation can be seen near 50 psec.

Momentum distributions along $p_{z}$ are obtained by rotating the GaAs switch by $90^{\circ}$. As anticipated for a pure radial wave packet, these distributions show the same oscillations (period and phase) as those observed in the $p_{x}$ distributions. Unfortunately, the geometry of the transient attenuator (see Fig. 1) reduces the field amplitude of s polarized HCP radiation (along $\hat{z}$ ) by approximately $40 \%$ relative to $p$-polarized (along $\hat{x}$ ) pulses. Therefore, there is insufficient field to saturate the ionization probability and no information on the probability distribution for momenta greater than 0.015 a.u. can be obtained. Nevertheless, we have observed the periodic breathing of the wave packet in this dimension as well.

A final test of the symmetry of the wave packet is performed by changing the polarity of the HCP in both the $\hat{x}$ and $\hat{z}$ orientations. Since a pure radial wave packet has no momentum asymmetry along any axis, the observed probability distributions are expected to be independent of the polarity of the HCP. Indeed, the momentum distributions taken along either the $\hat{x}$ or $\hat{z}$ axis are independent of the sign of the HCP field.

\section{ANALYSIS}

Because the radial wave packet under study is relatively well characterized, the measured probability distributions can be compared to theoretical expectations. The wave packet used in the quantum simulation is numerically prepared in the following manner. First, the magnitude of the probability amplitude for each state in the simulated wave packet is given by the measured Rydberg eigenstate distribution. Second, we assume that the laser pulse that excites the wave packet is transform limited so that the amplitude of each constituent eigenstate is real at the peak of the laser intensity at $t=0$. Next, the radial wave functions for the constituent eigenstates are calculated numerically using a $\mathrm{Nu}$ merov integration routine. Finally, the momentum space 
eigenfunctions are computed [16] and used to propagate the wave packet through time. The projections of the momentum space probability distribution along the $\hat{x}$ and $\hat{z}$ axes are then obtained from the time-dependent momentum space wave function. The calculated evolution of the probability distribution along $p_{x}$ is shown in Fig. 4. Figure 4(a) gives the distribution calculated with high temporal resolution, while Fig. 4(b) shows the same distribution averaged over the experimental resolution of approximately $1.5 \mathrm{psec}$.

There is qualitative agreement between the measured and calculated distributions in Figs. 3 and 4(b), respectively. The radial oscillations that are readily observable in both plots occur with the same period and phase. However, there are also clear differences between the measured and simulated results. First, the measured distribution shows a clear asymmetry about zero momentum even though the expectation value $\left\langle p_{x}\right\rangle \approx 0$ at all delays. Second, in the measured distribution, the times at which the wave packet has the largest probability of having a large positive momentum do not coincide precisely with the times at which the wave packet has the largest probability of having a large negative momentum. One would expect that when the wave packet is near the nucleus that it would simultaneously have its largest probability of having a large linear momentum in all directions.

Neither of these features appears in the simulation nor are they expected from a simple physical picture of the wavepacket motion. Nevertheless, they are robust and persist in the data for all HCP polarization directions and polarities. If for some electron velocity there was an actual difference in the probability for finding the electron moving in the positive rather negative direction, then the features in the momentum distribution would reverse under a change in the HCP polarity. Since no such reversals are observed, it is clear that primary differences in the data and simulation are not due to the properties of the wave packet, but instead are an artifact of the measurement technique.

The fundamental assumption of the IMR method is that the spatial probability distribution of the wave packet does not change during its interaction with the HCP. Clearly, this assumption is not valid at those times when the wave packet is traveling very rapidly near the nucleus. In this experiment, the duration of the HCP is approximately $1 / 5$ of the Kepler orbit period. However, a classical Rydberg electron with low angular momentum requires a time of only $1 / 10$ of the Kepler period to travel from the nucleus $(\approx 0$ a.u.) to a distance $n^{2}$ a.u. away (half the distance to the outer turning point). Therefore, it should not be surprising that the IMR method does not accurately portray the momentum distribution of the wave packet during those times when the wave packet is near the nucleus and its interaction with the HCP is nonimpulsive. In fact, due to the extremely high probability of finding the electron near the nucleus at certain times during its evolution, low angular momentum radial wave packets are probably the least well suited to characterization via IMR. Wave packets involving predominantly higher angular momentum states for which there is little probability of finding the electron near the nucleus are the best candidates for IMR [17]. Interestingly, it is these higher angular momentum wave packets that are the most difficult to probe using the more conventional techniques of ionization by short optical pulses [3] or bound-state interferometry [4].
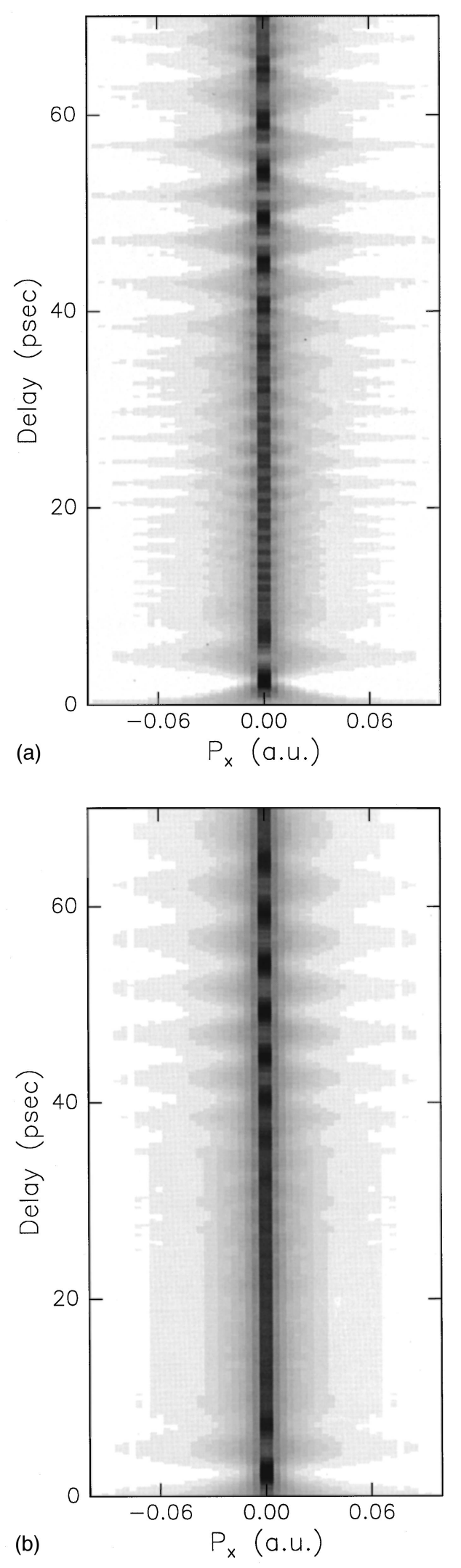

FIG. 4. Density plots analogous to those shown in Fig. 3 obtained from the quantum simulation described in the text. (a) shows the result of the calculation. (b) shows the result in (a) averaged through a 1.5-psec window to simulate the detector resolution of 1.5 psec for a more direct comparison with experiment. Note that the symmetry of the distribution is not reduced by poor temporal resolution. 
It is beyond the scope of this work to attempt a full quantum simulation that includes the effects of nonimpulsive ionization of the wave packet. However, since the IMR method is essentially classical in origin, we have simulated the wavepacket ionization classically in an attempt to reproduce the qualitative features of the observed momentum distributions. The simulation begins with an ensemble of electrons with a distribution of energies and well-defined angular momentum in a spherical shell near a hydrogenic nucleus. The discrete energy distribution is weighted according to the eigenstate distribution in the experimental wave packet. A fifth-order, adaptive-step Runge-Kutta algorithm integrates the classical equations of motion for the electron distribution. At some time after the distribution is launched from the nucleus, it is subjected to a Gaussian HCP. After the HCP, the fraction of the ensemble with positive energy gives the ionization probability. The procedure is repeated for another time delay and/or HCP field. The classical simulation produces a set of numerical data, wave-packet ionization probability versus delay versus HCP field, in complete analogy to the experiment. Simulated momentum distributions are derived from the calculated ionization probabilities using a procedure that is identical to the one discussed above for the treatment of the experimental data. The simulated distributions are averaged over a 1.5-psec window to account for the 0.5-psec duration of the actual wave packet and the resolution of the detector.

Figure 5(a) shows the results of the classical simulation using a HCP with a duration of 100-fsec while Fig. 5(b) shows the results obtain from a 1-psec pulse. The results obtained with the $100-$ fsec pulse show the same symmetric momentum oscillations that are seen in the quantum calculation. However, the 1-psec results have an asymmetry about zero momentum that is very similar to that seen in the experiment. The differences in the distributions produced using 100 -fsec and 1-psec pulses are due to two different classical effects. First, since the wave packet spends very little time moving at high velocity near the nucleus, its average rms velocity during the pulse is decreased. Therefore, the amplitudes of the positive and negative momentum wings in the apparent probability distribution are reduced. Since the actual momentum distribution is symmetric, this effect narrows the retrieved distribution symmetrically.

A second effect is responsible for the asymmetry observed in the distributions. When a low angular momentum electron passes the nucleus, its velocity changes sign. So for a certain electron velocity and HCP field strength, the momentum gained (lost) by the electron as it travels toward the nucleus can be exactly canceled by the momentum lost (gained) after reflecting from the nucleus. This impulse cancellation makes it very difficult or impossible to ionize a certain class of electrons in the classical ensemble that scatter from the nucleus during the HCP. However, it is precisely those electrons, moving at high velocity parallel or antiparallel to a true impulse that are the easiest and most difficult to ionize, respectively. Therefore, with a pulse of finite duration, the rising edge of the ionization versus field curve is pushed to higher fields relative to the impulsive ionization result and total saturation of that curve becomes more difficult or impossible. However, the ionization of those electrons in the distribution that are stationary or moving very
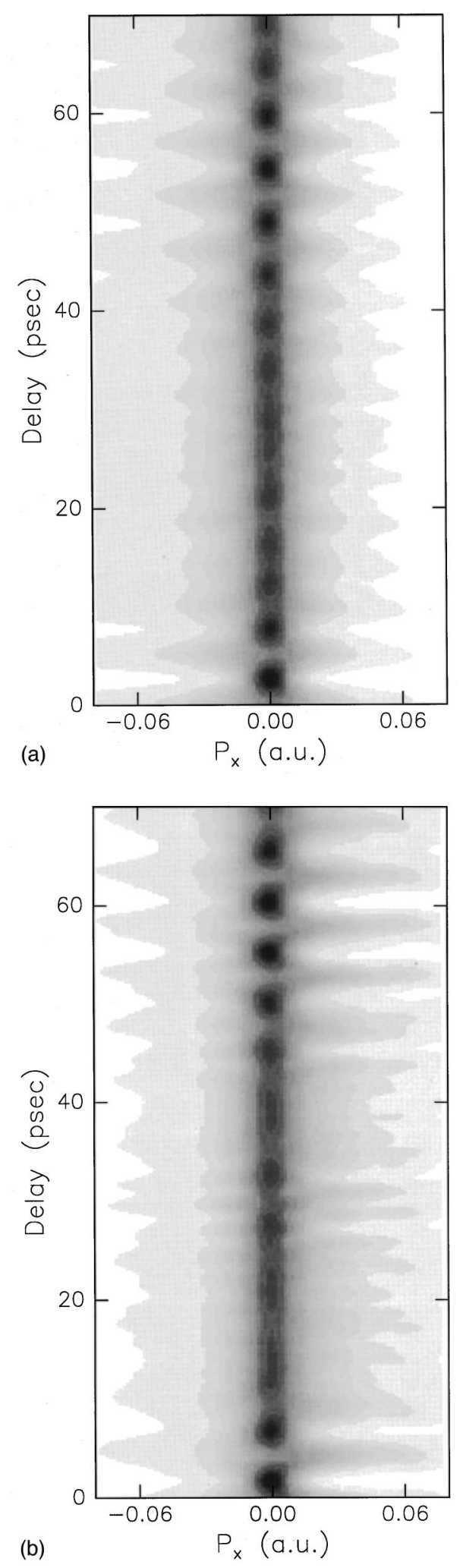

FIG. 5. Density plots of momentum distributions obtained by a classical simulation of the experiment. Ionization of a wave packet is calculated classically and the momentum distribution is obtained from the ionization curves as in the experiment. (a) shows the result of the calculation using a 0.1 -psec HCP while (b) shows the distribution obtained from a 1-psec HCP. Both (a) and (b) are temporally averaged with a 1.5-psec window to simulate the detector response. Note that an obvious asymmetry between the negative and positive wings of the distribution appears in (b) but not in (a). 
slowly during the pulse is not affected by an increase in the HCP duration, provided the pulse remains significantly shorter than the Kepler period. Therefore, the probability distribution, which is essentially a scaled derivative of the ionization probability curve, rises more sharply and falls less sharply than the true distribution, producing the observed asymmetry.

The source of the temporal shift of the large positive momentum maxima that is observed in the data is not obvious from the simulation. It may be due to nonhydrogenic core scattering or purely quantum effects. More probably, it is due to imperfect characterization of the HCP, which is certainly not Gaussian. Pulse shape effects combined with the nonimpulsive core scattering discussed in the preceding paragraph might produce a similar ionization artifact.

\section{DISCUSSION}

In previous work, IMR has been shown to be a potentially powerful measurement method $[8,18]$. It is particularly useful for monitoring the evolution of nonstationary wave packets far from the nucleus where optical frequency probe techniques fail $[1,2]$. However, this study clearly shows that care must be taken in interpreting the measured probability distribution if the impulse approximation is not strictly valid at all times during the wave packet's evolution. A breakdown in the impulse approximation does not simply reduce the resolution of the method through temporal blurring. It can also generate artificial asymmetries in the probability distributions. Nevertheless, the general behavior of even radial wave packets is clearly represented in the experimental measurement using 1-psec pulses.

Even considering its limitations, the IMR method provides considerably more information on the dynamic evolution of electron wave packets than either optical pump-probe ionization or bound-state interferometry. However, the IMR method is extremely data intensive. Therefore, without a high repetition rate system or single-shot detector, it is not feasible to implement the IMR technique to study wavepacket dynamics as a function of some external parameter. The single-shot detector allows one to acquire pump-probe data in real time reducing the time required to make the

[1] R. R. Jones and L. D. Noordam, Adv. At., Mol., Opt. Phys. 38, 1 (1997), and references therein.

[2] L. D. Noordam and R. R. Jones, J. Mod. Opt. 44, 2515 (1997).

[3] A. ten Wolde et al., Phys. Rev. Lett. 61, 2099 (1988); J. A. Yeazell, M. Mallalieu, and C. R. Stroud, Jr., ibid. 64, 2007 (1990).

[4] N. F. Scherer et al., J. Chem. Phys. 93, 856 (1990); L. D. Noordam, D. I. Duncan, and T. F. Gallagher, Phys. Rev. A 45, 4734 (1992); B. Broers et al., Phys. Rev. Lett. 71, 344 (1993); R. R. Jones, C. S. Raman, D. W. Schumacher, and P. H. Bucksbaum, ibid. 71, 2575 (1992); R. R. Jones, D. W. Schumacher, T. F. Gallagher, and P. H. Bucksbaum, J. Phys. B 28, 405 (1995).

[5] G. M. Lankhuijzen and L. D. Noordam, Phys. Rev. Lett. 76, 1784 (1996). measurements by one to two orders of magnitude. A full set of ionization versus delay versus HCP field can be obtained in minutes, essentially removing difficulties due to slow equipment drifts. Furthermore, the single-shot capability provides real-time feedback for adjusting equipment, making it possible to tune the wave packet to have specific temporal properties [19].

Measurement of all three Cartesian components of the momentum distribution of hydrogenic wave packets has an interesting benefit that should be noted. In IMR, the probability distribution, and not the momentum space wave function, is measured. Therefore, the spatial probability distribution cannot be recovered. However, this does not imply that no information regarding the position of the wave packet can be obtained. In fact, the time-dependent expectation value $\langle 1 / r\rangle$ is easily retrieved if all three Cartesian components of the momentum distribution have been measured. This expectation value gives important information on the proximity of the wave packet to the nucleus. Using the measured distributions, the expectation values $\left\langle p_{x, y, z}^{2}\right\rangle$ can be computed and inserted into the nonrelativistic hydrogenic Hamiltonian

$$
\langle H\rangle=\frac{-1}{2 N^{2}}=\frac{1}{2}\left(\left\langle p_{x}^{2}\right\rangle+\left\langle p_{y}^{2}\right\rangle+\left\langle p_{z}^{2}\right\rangle\right)-\langle 1 / r\rangle \text { a.u. }
$$

to give the desired result. Unfortunately, this determination is extremely sensitive to the amplitude of the far wings of the momentum distribution, which decreases polynomially and not exponentially. Therefore, computing $\left\langle p^{2}\right\rangle$ requires a very accurate determination of the HCP ionization probability near $0 \%$ and $100 \%$ as well as an extremely short pulse to ensure that the impulse approximation is valid at high electron momentum. While neither of these criteria is well satisfied in the current experiment, future experiments might take advantage of the relation in Eq. (1). It is clear that a new source of ultrashort, high-field HCPs would be of great utility for probing electronic wave packets.

\section{ACKNOWLEDGMENTS}

We gratefully acknowledge the support of the AFOSR and the Packard Foundation.

[6] J. G. Story, D. I. Duncan, and T. F. Gallagher, Phys. Rev. Lett. 71, 3431 (1993).

[7] R. R. Jones, Phys. Rev. A 57, 446 (1998).

[8] R. R. Jones, Phys. Rev. Lett. 76, 3927 (1996).

[9] M. B. Campbell, T. J. Bensky, and R. R. Jones, Optics Express 1, 197 (1997).

[10] T. F. Gallagher, Rydberg Atoms, 1st ed. (Cambridge University Press, Cambridge, 1994).

[11] D. You, R. R. Jones, D. R. Dykaar, and P. H. Bucksbaum, Opt. Lett. 18, 290 (1993).

[12] N. E. Tielking, T. J. Bensky, and R. R. Jones, Phys. Rev. A 51, 3370 (1995).

[13] R. R. Jones, D. You, and P. H. Bucksbaum, Phys. Rev. Lett. 70, 1236 (1993).

[14] C. Raman, C. W. S. Conover, C. I. Sukenik, and P. H. Bucks- 
baum, Phys. Rev. Lett. 76, 2436 (1996); M. T. Frey, F. B. Dunning, C. O. Reinhold, and J. Burgdorfer, Phys. Rev. A 55, R865 (1997); C. Raman, T. C. Weinacht, and P. H. Bucksbaum, ibid. 55, R3995 (1997).

[15] C. O. Reinhold, H. Shao, and J. Burgdorfer, J. Phys. B 27, L469 (1994); C. O. Reinhold, M. Melles, H. Shao, and J. Burgdorfer, ibid. 26, L659 (1993); M. T. Frey, F. B. Dunning, C. O. Reinhold, and J. Burgdorfer, Phys. Rev. A 53, R2929 (1996); C. O. Reinhold, J. Burgdorfer, M. T. Frey, and F. B. Dunning, ibid. 54, R33 (1996).
[16] B. Podolsky and L. Pauling, Phys. Rev. 34, 109 (1929).

[17] Examples are Stark wave packets such as those studied by Broers et al. (see Ref. [4]) or linear wave packets created with HCPs (see Ref. [8]).

[18] F. Robicheaux, Phys. Rev. A 56, R3358 (1997).

[19] The single-shot detector can be used in virtually any type of pump-probe experiment. By increasing the angle between the direction of propagation of the pump and probe beams from near 0 to $\pi$, the temporal resolution of the detector can be adjusted from subfemtosecond to picosecond as required for a particular experiment. 